\title{
P04.78. Development of an integrative service model for dysthymia patients with body-mind- spirit approach in Chinese medicine clinics in Hong Kong
}

\author{
M Li ${ }^{* *}$, C Chan ${ }^{2}$, C Chan ${ }^{2}$, H Chan ${ }^{2}$, L Hui ${ }^{1}$, E Ziea ${ }^{1}$ \\ From International Research Congress on Integrative Medicine and Health 2012 \\ Portland, Oregon, USA. 15-18 May 2012
}

\section{Purpose}

The prevalence of anxiety and mood disorders in Hong Kong was found to be $4.1 \%$ and $8.4 \%$ respectively. This study aimed at exploring a sustainable and practical model for incorporation of patient empowerment element, through an integrative Body-Mind-Spirit (I-BMS) approach, for treatment of dysthymia patients in Chinese Medicine (CM) out-patient clinics in Hong Kong.

\section{Methods}

In the first pilot, in addition to routine CM treatment (herbal and acupuncture), CM Practitioners also provided general psychological counseling, advice on dietary regime and self-administered acupressure based on syndrome differentiation for dysthymia. The Centre on Behavioral Health at the University of Hong Kong was commissioned in the second phase to develop a tailormade I-BMS intervention program with CM concepts. The Centre provided six 3-hours sessions of I-BMS intervention for dysthymia patients recruited. Evaluation included validated questionnaires like the Hospital Anxiety and Depression Scale (HADS) and the Brief Symptom Inventory 18 (BSI-18) for pre-post comparison of clinical outcomes.

\section{Results}

Sixty-six patients participated in the initial pilot and the major CM diagnosis was $\mathrm{Bu}$ Mi (insomnia). Fifty-eight patients attended the group intervention sessions in phase II with average attendance rate of $91.8 \%$. Among those who completed the HADS and BSI-18 questionnaires $(n=45)$, there was a significant drop $(\mathrm{p}<0.01)$ in domains of anxiety and depression in HADS and BSI scores, which indicated clinical improvement.

\section{Conclusion}

Given resource and manpower considerations in $\mathrm{CM}$ clinics, the patient empowerment model in phase II was clinically practical and effective, fostering a synergic effect with CM treatment. The way forward is to integrate I-BMS patient empowerment element into CM service for dysthymia treatment in a "train the trainer" approach. Content of the I-BMS intervention will be consolidated to produce a trainer's manual for CMPs and a set of patient empowerment material. The resulting service model will be led by CMPs equipped with IBMS knowledge and skills.

\section{Author details \\ ${ }^{1}$ Chinese Medicine Department, Hospital Authority, Hong Kong, Hong Kong. ${ }^{2}$ The Centre on Behavioral Health, the University of Hong Kong, Hong Kong, Hong Kong.}

Published: 12 June 2012 